\title{
Electron radiation damage mechanisms in 2D $\mathrm{MoSe}_{2}$
}

\author{
T. Lehnert, ${ }^{1, \text { a) }}$ O.Lehtinen, ${ }^{1}$ G. Algara-Siller, ${ }^{2, b)}$ and U. Kaiser ${ }^{2}$ \\ 1) Central Facility for Electron Microscopy, Group of Electron Microscopy of Materials Science, Ulm University, \\ Ulm 89081, Germany \\ ${ }^{2)}$ Central Facility for Electron Microscopy, Group of Electron Microscopy of Materials Science, Ulm University, \\ Germany
}

The contributions of different damage mechanisms in single-layer $\mathrm{MoSe}_{2}$ were studied by investigating different $\mathrm{MoSe}_{2}$ /graphene heterostructures by aberration-corrected high-resolution transmission electron microscopy (AC-HRTEM) at $80 \mathrm{keV}$. The damage cross-sections were determined by direct counting of atoms in the AC-HRTEM images. The contributions of damage mechanisms such as knock-on damage or ionization effects were estimated by comparing the damage rates in different heterostructure configurations, similarly to what has been earlier done with $\mathrm{MoS}_{2}$. The behaviour of $\mathrm{MoSe}_{2}$ was found to be nearly identical to that of $\mathrm{MoS}_{2}$, which is an unexpected result, as the knock-on mechanism should be suppressed in MoSe 2 due to the high mass of Se as compared to S.

PACS numbers: Valid PACS appear here

Keywords: Suggested keywords

\section{INTRODUCTION}

Modern transmission electron microscopes (TEM) allow imaging materials at a single atom resolution with acceleration voltages in the range of $20-300 \mathrm{kV}^{1-3}$, foremost thanks to the practical realization of aberration correctors (AC) for transmission electron microscopy ${ }^{4,5}$. However, high electron doses are required for achieving a high enough signal to noise ratio for accurate detection of the atom position in the image. This sets strong requirements on the studied materials in terms of radiation damage resistance, and in practice many materials are destroyed by the interaction with the electrons before a clear image can be acquired. For controlling the radiation damage, that is, for finding the optimal experimental conditions for each material, it is essential to understand the underlying damage mechanisms.

Radiation damage in a TEM is often split into two categories. First, elastic damage, or knock-on damage describes the processes involving direct elastic collisions between the imaging electrons and the target atoms. Upon each collision, momentum is transferred to the target atom, and if the momentum exceeds a material dependent critical limit, the atom is displaced from its original position. This limit in transferred momentum or kinetic energy leads into a threshold electron energy, below which knock-on damage should not occur. Knock-on damage is the most studied mechanism ${ }^{6-10}$, mainly due to the simplicity of the process as compared to the other mechanisms. Recent experiments have shown, however, that there is no sharp cut off in knock-on damage due to thermal vibrations of the target atoms, and a finite displacement probability remains below the static thresh-

\footnotetext{
a) tibor.lehnert@uni-ulm.de

b) Now at Department of Inorganic Chemistry, Fritz-Haber-Institut der Max-Planck-Gesellschaft, Berlin 14195, Germany
}

old $^{11}$. Even taking this effect into account, the knockon cross section becomes vanishingly small far below the static threshold.

The second is a broader category grouping together all the inelastic processes, where the imaging electrons interact with the electronic system of the target, weakening or breaking bonds, heating the material and/or accumulating electrostatic charge ${ }^{12,13}$. However, direct heating of the sample by the electron beam has been estimated to be minor ${ }^{14}$, especially in a $2 \mathrm{D}$ material such as $\mathrm{MoS}_{2}$ and $\mathrm{MoSe}_{2}$.

Damage can occur also via indirect routes, such as chemical etching, where the electrons break down residual impurities such as oxygen molecules or water in the microscope, and the formed radicals in turn attack the sample material causing its damage.

Recently, an experimental scheme for separating the contributions of different damage mechanisms in singlelayer $\mathrm{MoS}_{2}$ was developed ${ }^{15}$. In the experiments $\mathrm{MoS}_{2}$ /graphene heterostructures were investigated by HRTEM, and by comparing the damage rates in different sample geometries, estimates on the different damage mechanism contributions were attained.

The following comparisons were used ${ }^{15}$. 1) The difference between the damage cross section in a sample with the entry surface covered with graphene $\left(\mathrm{G} / \mathrm{MoS}_{2}\right)$ and a sample with the exit surface covered $\left(\mathrm{MoS}_{2} / \mathrm{G}\right)$ gives the knock-on contribution. This lays on the assumption that only the knock-on process has a directional dependence, that is, if the entry surface is covered, the sulfur atoms at the bottom can still be displaced into the vacuum (knock-on active), where as with covering the exit surface the displaced sulfur atoms are stopped by the graphene layer (knock-on disabled). 2) The difference between a free-standing $\mathrm{MoS}_{2}$ and $\mathrm{G} / \mathrm{MoS}_{2}$ gives the inelastic contribution. This, in turn, is based on the assumption that graphene, which is a supreme electric and thermal conductor, quenches the electronic excitations and dissipates any introduced heat and eliminates 
sample charging, thus removing the inelastic damage ${ }^{16}$. Of the two, the latter is more conjecture because the damage created by inelastic collisions with high energy electrons is not fully understood.

Here, to extend on the earlier study with $\mathrm{MoS}_{2}$, similar experiments were conducted with $\mathrm{MoSe}_{2}$ /graphene heterostructures. $\mathrm{MoS}_{2}$ and $\mathrm{MoSe}_{2}$ have identical crystal structures and they are isoelectronic ${ }^{17-19}$, while the main difference is the different masses of the chalcogen atoms (32.07 amu for S, and $79.0 \mathrm{amu}$ for Se) and the higher amount of electrons (34 for Se, and 16 for S) which increases the probability for electron excitations due to the electron beam. As the momentum transfer from the impinging electrons to the target atoms is dependent on the target atom mass, knock-on damage can be expected to be suppressed in the case of $\mathrm{MoSe}_{2}$ with $80 \mathrm{keV}$ electrons (the minimum threshold energy is $6.4 \mathrm{eV}$ for $\mathrm{MoSe}_{2}$ and $6.9 \mathrm{eV}$ for $\mathrm{MoS}_{2}$ which corresponds, due to the different masses, to a static threshold electron energy of $190 \mathrm{keV}$ and $90 \mathrm{keV}$ respectively ${ }^{6}$ ). Furthermore, the knock-on threshold for the chalcogen atoms in the upper layer is lower because they are stopped by the subjacent layers ${ }^{6}$. On the other hand the formation energy of chalcogen vacancy in $\mathrm{MoSe}_{2}$ is $6.1 \mathrm{eV}$ as compared to $6.7 \mathrm{eV}$ in $\mathrm{MoS}_{2}$, which can be reflected as incresed vacancy yield through inelastic processes ${ }^{6}$. Suprisingly, $\mathrm{MoSe}_{2}$ has almost identical behaviour to $\mathrm{MoS}_{2}$ in our experiment. This result calls for critical assessment of the assumptions about the damage mechanisms, especially the strict separation into the elastic and inelastic categories, and the experimental scheme employed here for the separation of the different contributions.

\section{RESULTS AND DISCUSSION}

The heterostructure samples were constructed from mechanically exfoliated flakes on $\mathrm{SiO}_{2}$. In the preparation process, Au Quantifoil TEM grids were placed on top of the $\mathrm{MoSe}_{2}$ or graphene flakes with the position precisely controlled by a micromanipulator. The Quantifoil film was brought into contact by evaporating isopropyl alcohol after which the $\mathrm{SiO}_{2}$ was etched with $\mathrm{KOH}$, releasing the grid with the flake. The process was repeated to bring more flakes on top of the previously transferred one(s), resulting in the desired heterostructures.

Based on optical microscopy images, such as shown in Figure 1a, the heterostructure was located in the TEM. Electron diffraction patterns were recorded to confirm the presence of the required layers (see Figure $1 \mathrm{~b}$ for an example of $\mathrm{G} / \mathrm{MoSe}_{2} / \mathrm{G}$ ) and the monolayer nature of the $\mathrm{MoSe}_{2}$ flake $^{20}$.

Once the correct flake was located, AC-HRTEM image sequences were acquired at high magnification to be able to directly count the produced vacancies. The electron dose rate was in the order of $10^{6} \mathrm{e} /\left(\mathrm{nm}^{2} \mathrm{~s}\right)$ and a vacuum level was in the order of $10^{-8}$ mbar during the experiment.

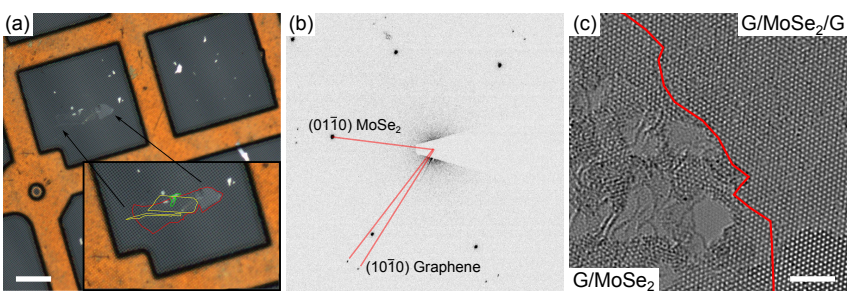

FIG. 1. (a) An optical microscopy image from a quantifoil $\mathrm{Au}$-grid with a $\mathrm{G} / \mathrm{MoSe}_{2} / \mathrm{G}$ sandwich. The different layers are enlarged in the inset and framed with colors, where the red and yellow flakes are graphene and the green one is $\mathrm{MoSe}_{2}$. The scale bar corresponds to $50 \mu \mathrm{m}$. (b) An electron diffraction pattern of $\mathrm{G} / \mathrm{MoSe}_{2} / \mathrm{G}$ with the $\mathrm{MoSe}_{2}$ peaks at $3.5 \mathrm{~nm}^{-1}$ and graphene peaks at $4.7 \mathrm{~nm}^{-1}$. The neighbouring graphene peaks correspond to two graphene layers which are in slightly different orientations. (c) A $80 \mathrm{kV}$ AC-HRTEM image of $\mathrm{G} / \mathrm{MoSe}_{2}$ (left area) and $\mathrm{G} / \mathrm{MoSe}_{2} / \mathrm{G}$ (right area) after a total electron dose of $3.1 \times 10^{9} \mathrm{e} / \mathrm{nm}^{2}$. It is striking that the left area with the $\mathrm{G} / \mathrm{MoSe}_{2}$ configuration is highly damaged while the right area is still in a good condition. The scale bar corresponds to $3 \mathrm{~nm}$.

The effect of the graphene coating is evident in Figure $1 \mathrm{c}$, where the edge of the bottom graphene layer runs through the field of view (that is, on the left there is a $\mathrm{G} / \mathrm{MoSe}_{2}$ structure and on the right $\mathrm{G} / \mathrm{MoSe}_{2} / \mathrm{G}$ structure). After an electron dose of $3 . \times 10^{9} \mathrm{e} / \mathrm{nm}^{2}$ the $\mathrm{G} / \mathrm{MoSe}_{2}$ is heavily damaged, while on the $\mathrm{G} / \mathrm{MoSe}_{2} / \mathrm{G}$ the structure is still relatively intact.

Figure 2 shows the qualitative difference between the four sample configurations. For every configuration, an initial HRTEM image and an image after a dose of $\phi=7.7 \times 10^{8} \mathrm{e}^{-} / \mathrm{nm}^{2}$ are shown. In the case of the free-standing $\mathrm{MoSe}_{2}$ (fig. 2(a)), the sample was heavily damaged by the dose with extensive disorder and a large hole visible in the image. The images of $\mathrm{G} / \mathrm{MoSe}_{2}$ (fig. 2(b)) show reduced damage but the $\mathrm{MoSe}_{2}$ layer is still visibly damaged. The damage in $\mathrm{MoSe}_{2} / \mathrm{G}$ is further reduced (fig. 2(c)). The highest improvement in radiation resistance is found in the $\mathrm{G} / \mathrm{MoSe}_{2} / \mathrm{G}$ case: in figure 2(d) it can be seen that the vacancy concentration remains unchanged after a dose which destroyed the free-standing $\mathrm{MoSe}_{2}$, although migration of vacancies is taking place also in this configuration.

For determining the vacancy production cross sections for each heterostructure, frames from the beginning of the image sequence and at a maximum vacancy concentration of approximately $7 \%$ were compared, and the total number of new vacancies was directly counted. Due to the stacked structure of Mo atoms between Se in a monolayer, only Se vacancies are produced by the electron beam. As the electron dose between these frames is known, the probability of creating a vacancy per electron impact (i.e., the cross section $\sigma$ ) can be calculated by $\Delta \mathrm{V} /(\mathrm{N} \phi)$, where $\Delta \mathrm{V}$ is the number of new vacancies, $\mathrm{N}$ the total number of Se sites within the analyzed area, and $\phi$ the electron dose in $\mathrm{e} / \mathrm{nm}^{2}$. 

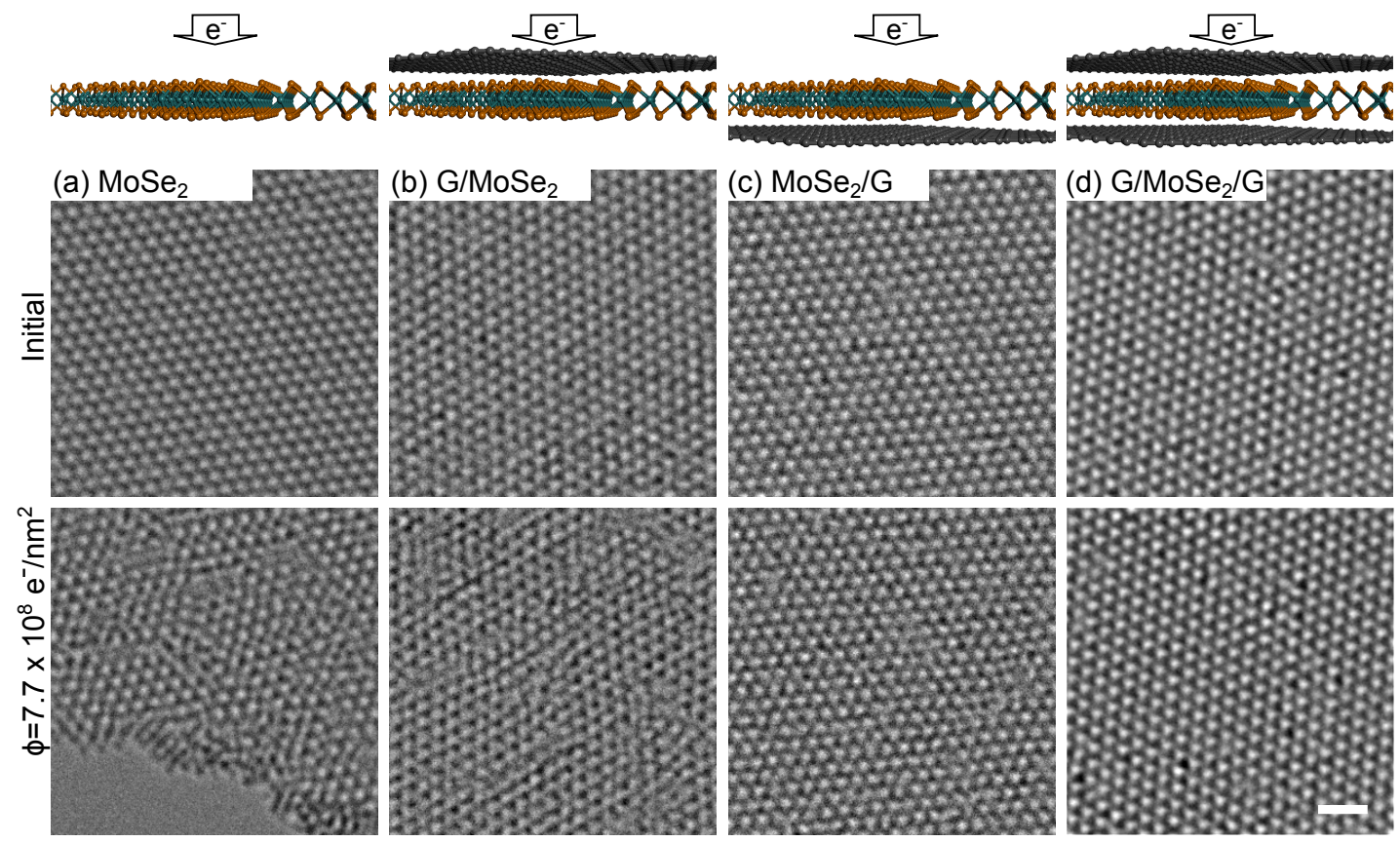

FIG. 2. Comparison of the damage rate on TMDs under the electron beam for different heterostructure configurations. The initial HRTEM image, and an image after an dose of $\phi=7.7 \times 10^{8} \mathrm{e}^{-} / \mathrm{nm}^{2}$ are shown. (a) Free-standing $\mathrm{MoSe}_{2}$ (b) MoSe $\mathrm{M}_{2}$ with a protection layer of graphene on the entry surface of the electron beam $\left(\mathrm{G} / \mathrm{MoSe}_{2}\right)$. (c) MoSe $\mathrm{C}_{2}$ with graphene on the exit surface $\left(\mathrm{MoSe}_{2} / \mathrm{G}\right)$. (d) A Sandwich where MoSe 2 was coated with graphene from both sides (G/MoSe $\left.2 / G\right)$. The HRTEM images (a-d) show an increase in resistance against radiation damage going from free-standing to the G/MoSe $2 / G$ heterostructure. The scale bar corresponds to $1 \mathrm{~nm}$. The frames were numerically corrected for residual $\mathrm{A}_{2}$ astigmatism in the order of $100 \mathrm{~nm}{ }^{21}$.

Quantification of the damage rates is summarized in Table I, and the numbers agree with the previous visual assessment. The cross sections for the $\mathrm{MoSe}_{2}, \mathrm{G} / \mathrm{MoSe}_{2}$, $\mathrm{MoSe}_{2} / \mathrm{G}$, and $\mathrm{G} / \mathrm{MoSe}_{2} / \mathrm{G}$ configurations are determined to be 4.1(4) b, 1.5(2) b, 0.44(4) b, and 0.011(4) $\mathrm{b}$, respectively, where $\mathrm{b}$ stands for barn $\left(10^{-38} \mathrm{~m}^{2}\right)$ and the number in parenthesis is the confidence interval of the last digits. In earlier studies, a damage cross section of $3.9 \mathrm{~b}$ for free-standing $\mathrm{MoSe}_{2}{ }^{10}$ was found, which is in good agreement with our value. Compared to the free-standing case, the damage resistance is increased by factors of 2.7, 9.3 and $\sim 400$ for the G/MoSe $2, \mathrm{MoSe}_{2} / \mathrm{G}$, and $\mathrm{G} / \mathrm{MoSe}_{2} / \mathrm{G}$, respectively. Damage in the graphene layers was not evaluated, but earlier studies have shown graphene to be stable under an electron beam of $80 \mathrm{keV}^{9}$.

Assuming that only the knock-on process depends on the direction of the electron beam, the knock-on cross section can be calculated as the difference between the two cross sections, yielding 1.0(2) b. This accounts for $24 \%$ of the total vacancy production cross section in the free-standing case.

The inelastic contribution can be estimated by assuming that already coating a single side of $\mathrm{MoSe}_{2}$ with graphene suppresses this damage channel due to the quick dissipation of heat, charge, and electronic excitations by the graphene layer. The difference between the cross sections for the $\mathrm{MoSe}_{2}$ and $\mathrm{G} / \mathrm{MoSe}_{2}$ cases yields $2.6(5)$ as the inelastic cross section, accounting for $63 \%$
TABLE I. Quantitative results for the four different heterostructure configurations. $\mathrm{N}$ is the total number of the selenium atoms in the investigated area and $\mathrm{V}$ is the number of the produced vacancies after an dose $\phi$. The damage-crosssection $\sigma$, which describes the vacancy production probability, is determined with $\Delta \mathrm{V} /(\mathrm{N} \phi)$. For the confidence intervals we took $\sqrt{\mathrm{N}}$ for $\mathrm{N}, \sqrt{\mathrm{V}}$ for $\mathrm{V}$, and $1 \%$ for the electron dose were assumed. The values for the damage-cross-section of $\mathrm{MoS}_{2}$ were taken from ${ }^{15}$.

\begin{tabular}{|c|c|c|c|c||c|}
\hline Configuration & $\mathrm{N}$ & $\Delta \mathrm{V}$ & $\phi\left(\mathrm{e} / \mathrm{nm}^{2}\right)$ & $\sigma(\mathrm{b})$ & $\sigma(\mathrm{b})$ for $\mathrm{MoS}_{2}$ \\
\hline $\mathrm{MoSe}_{2}$ & 4305 & 133 & $7.5 \times 10^{7}$ & $4.1(4)$ & $4.5(4)$ \\
\hline $\mathrm{G} / \mathrm{MoSe}_{2}$ & 4032 & 87 & $1.4 \times 10^{8}$ & $1.5(2)$ & $1.5(2)$ \\
\hline $\mathrm{MoSe}_{2} / \mathrm{G}$ & 4799 & 114 & $5.4 \times 10^{8}$ & $0.44(5)$ & $0.48(4)$ \\
\hline $\mathrm{G}_{\mathrm{MoSe}} / \mathrm{G}$ & 2908 & 8 & $2.7 \times 10^{9}$ & $0.011(4)$ & $0.008(3)$ \\
\hline
\end{tabular}

of the total damage production.

Taking into account that $24 \%$ of the damage is produced by knock-on damage, $63 \%$ by heat, charge and/or electronic excitations, then $13 \%$ of the damage has to be produced by other mechanisms, such as chemical etching and further ionization effects.

The estimated significant contribution of knock-on damage brings the earlier conclusions ${ }^{15}$ into question, as the theoretical prediction would indicate no knockon damage ${ }^{6}$. Here, two possible explanations for this discrepancy can be offered.

First, one has to ask whether the methodology employed here is solid. More precisely, does the difference 
between the $\mathrm{G} / \mathrm{MoSe}_{2}$ and $\mathrm{MoSe}_{2} / \mathrm{G}$ cases really give the knock-on cross section? As there is, no other method available for isolating the knock-on contribution, the correctness of the method cannot be externally evaluated.

The alternative interpretation would indicate a marked shortcoming of the damage model where the damage mechanisms are simply divided into the elastic and inelastic contributions. For example, a process where knockon thresholds are influenced by inelastic scattering events prior to an electron impact, could play a significant role.

Two things can, however, be concluded from the experiment. First, there is a directional dependence in the damage process, based on the difference between the $\mathrm{G} / \mathrm{MoSe}_{2}$ and $\mathrm{MoSe}_{2} / \mathrm{G}$ cases, even if the exact damage mechanism cannot be determined. The damage rate is more strongly influenced when the exit surface of the sample is covered with graphene, which implicates faster damage rates on the exit surface. As the direction of the electron beam is the only difference between the surfaces, the directional dependence is clear.

The second conclusion, related to a comparison to the earlier $\mathrm{MoS}_{2}$ results (given in the last column of Table $\mathrm{I}^{15}$ ), is that the mass of the chalcogen atom does not seem to play a large role in the damage rates. Once again, the more than two-fold difference between the masses of $\mathrm{S}$ and Se allow one to expect a marked difference in the elastic damage. As the difference between the cross sections for $\mathrm{MoS}_{2}$ and $\mathrm{MoSe}_{2}$ is small or non-existent, taking the confidence intervals into account, one could conclude that the damage occurs via the inelastic route. However, no clear explanation for the directional dependence within the inelastic damage processes can be found here, which in turn would imply a contribution from elastic collisions between the electrons and the atoms. Expanding on the earlier suggestion of a two-step process, where inelastic processes first lower locally the knock-on threshold, after which another electron displaces the atom, it can be conjectured, that if the threshold becomes low enough, the knock-on cross-section becomes very high, meaning that the first inelastic step would become the rate limiting process, removing the mass dependence of the rate.

\section{CONCLUSIONS}

Electron beam-induced damage mechanisms were studied in single-layer $2 \mathrm{D} \mathrm{MoSe}_{2}$ in an aberrationcorrected high-resolution transmission electron microscope operated at $80 \mathrm{kV}$. The approach of the study was to construct different graphene-MoSe $\mathrm{M}_{2}$ heterostructures, which allow the control over different damage mechanisms under the electron beam, similar to what was done earlier with $\mathrm{MoS}_{2}{ }^{15}$. A significant contribution from the elastic (knock-on) damage was found, which is a surprising result, as the high mass of the Se atoms is expected to suppress the knock-on damage process at $80 \mathrm{kV}^{6}$. Comparing to the earlier results on $\mathrm{MoS}_{2}$, it was observed that the mass of the chalcogen atom does not influence the damage rates. However, a directional dependence of the damage process was found. Our explanation for the results presented here is a two-step process, where first an inelastic event significantly lowers locally the knockon threshold, after which a second electron displaces the atom via an elastic collision. These results make the need for further studies on the damage mechanisms clear, like the development of an external test for the method employed here to separating the damage mechanisms.

\section{ACKNOWLEDGEMENT}

The authors acknowledge financial support by the $\mathrm{EU}$ in the frame of the Graphene Flagship and by the DFG in the frame of the SALVE (Sub Angstrom LowVoltage Electron microscopy) project as well the Ministry of Science, Research and the Arts (MWK) of BadenWuerttemberg in the frame of the SALVE project.

${ }^{1}$ U. Kaiser, J. Biskupek, J. Meyer, J. Leschner, L. Lechner, H. Rose, M. Stöger-Pollach, A. Khlobystov, P. Hartel, H. Müller, M. Haider, S. Eyhusen, and G. Benner, Ultramicroscopy 111, 1239 (2011).

${ }^{2}$ O. L. Krivanek, N. Dellby, M. F. Murfitt, M. F. Chisholm, T. J. Pennycook, K. Suenaga, and V. Nicolosi, Ultramicroscopy 110, 935 (2010).

${ }^{3}$ M. Linck, P. Hartel, S. Uhlemann, F. Kahl, H. Müller, J. Zach, M. Haider, M. Niestadt, M. Bischoff, J. Biskupek, Z. Lee, T. Lehnert, F. Börrnert, H. Rose, and U. Kaiser, Phys. Rev. Lett. (2016).

${ }^{4}$ M. Haider, H. Rose, S. Uhlemann, E. Schwan, B. Kabius, and K. Urban, Ultramicroscopy 75, 53 (1998).

${ }^{5}$ O. L. Krivanek, M. F. Chisholm, V. Nicolosi, T. J. Pennycook, G. J. Corbin, N. Dellby, M. F. Murfitt, C. S. Own, Z. S. Szilagyi, M. P. Oxley, et al., Nature 464, 571 (2010).

${ }^{6}$ H.-P. Komsa, J. Kotakoski, S. Kurasch, O. Lehtinen, U. Kaiser, and A. V. Krasheninnikov, Phys. Rev. Lett. 109, 035503 (2012).

${ }^{7}$ J. Kotakoski, C. H. Jin, O. Lehtinen, K. Suenaga, and A. V. Krasheninnikov, Phys. Rev. B 82, 113404 (2010).

${ }^{8}$ J. C. Meyer, A. Chuvilin, G. Algara-Siller, J. Biskupek, and U. Kaiser, Nano Letters 9, 2683 (2009), pMID: 19480400, http://dx.doi.org/10.1021/nl9011497.

${ }^{9}$ J. C. Meyer, F. Eder, S. Kurasch, V. Skakalova, J. Kotakoski, H. J. Park, S. Roth, A. Chuvilin, S. Eyhusen, G. Benner, A. V. Krasheninnikov, and U. Kaiser, Phys. Rev. Lett. 108, 196102 (2012).

${ }^{10}$ O. Lehtinen, H.-P. Komsa, A. Pulkin, M. B. Whitwick, M.-W. Chen, T. Lehnert, M. J. Mohn, O. V. Yazyev, A. Kis, U. Kaiser, and A. V. Krasheninnikov, ACS Nano 9, 3274 (2015), pMID: 25748134, http://dx.doi.org/10.1021/acsnano.5b00410.

${ }^{11}$ J. C. Meyer, F. Eder, S. Kurasch, V. Skakalova, J. Kotakoski, H. J. Park, S. Roth, A. Chuvilin, S. Eyhusen, G. Benner, A. V. Krasheninnikov, and U. Kaiser, Phys. Rev. Lett. 108, 196102 (2012).

${ }^{12}$ R. Egerton, Ultramicroscopy 127, 100 (2013), frontiers of Electron Microscopy in Materials Science.

${ }^{13}$ X. Wei, D.-M. Tang, Q. Chen, Y. Bando, and D. Golberg, ACS Nano 7, 3491 (2013), pMID: 23458341, http://dx.doi.org/10.1021/nn400423y.

${ }^{14}$ R. Egerton, P. Li, and M. Malac, Micron 35, 399 (2004), international Wuhan Symposium on Advanced Electron Microscopy.

${ }^{15}$ G. Algara-Siller, S. Kurasch, M. Sedighi, O. Lehtinen, and U. Kaiser, Applied Physics Letters 103, 203107 (2013).

${ }^{16}$ O. Cretu, Y.-C. Lin, and K. Suenaga, Micron 72, 21 (2015). 
${ }^{17}$ S. Tongay, J. Zhou, C. Ataca, K. Lo, T. S. Matthews, J. Li, J. C. Grossman, and J. Wu, Nano Letters 12, 5576 (2012), pMID: 23098085, http://dx.doi.org/10.1021/nl302584w.

${ }^{18}$ M. Chhowalla, H. S. Shin, G. Eda, L.-J. Li, K. P. Loh, and H. Zhang, Nature chemistry 5, 263 (2013).

${ }^{19}$ J. He, K. Hummer, and C. Franchini, Phys. Rev. B 89, 075409 (2014)
${ }^{20}$ J. Brivio, D. T. L. Alexander, and A. Kis, Nano Letters 11, 5148 (2011), pMID: 22010987, http://dx.doi.org/10.1021/nl2022288.

${ }^{21}$ O. Lehtinen, D. Geiger, Z. Lee, M. B. Whitwick, M.-W. Chen, A. Kis, and U. Kaiser, Ultramicroscopy (2014). 